\title{
A workflow management system for the OR based on the OMG standards BPMN, CMMN, and DMN
}

M. Wiemuth, O. Burgert

M. Wiemuth, O. Burgert, "A workflow management system for the OR based on the OMG standards BPMN, CMMN, and DMN," Proc. SPIE 10951, Medical Imaging 2019: Image-Guided Procedures, Robotic Interventions, and Modeling, 1095127 (8 March 2019); doi: 10.1117/12.2512724

SPIE. Event: SPIE Medical Imaging, 2019, San Diego, California, United States 


\title{
A workflow management system for the OR based on the OMG standards BPMN, CMMN and DMN
}

\author{
M. Wiemuth ${ }^{a}$, O. Burgert ${ }^{a}$ \\ ${ }^{a}$ Reutlingen University, School of Informatics, Research Group Computer Assisted Medicine \\ (CaMed) Reutlingen, Germany \\ Contact: \{markus.wiemuth, oliver.burgert\}@ reutlingen-university.de
}

\begin{abstract}
Workflow driven support systems in the peri-operative area have the potential to optimize clinical processes and to allow new situation-adaptive support systems. We started to develop a workflow management system supporting all involved actors in the operating theatre with the goal to synchronize the tasks of the different stakeholders by giving relevant information to the right team members. Using the OMG standards BPMN, CMMN and DMN gives us the opportunity to bring established methods from other industries into the medical field.

The system shows each addressed actor their information in the right place at the right time to make sure every member can execute their task in time to ensure a smooth workflow. The system has the overall view of all tasks. Accordingly, a workflow management system including the Camunda BPM workflow engine to run the models, and a middleware to connect different systems to the workflow engine and some graphical user interfaces to show necessary information or to interact with the system are used. The complete pipeline is implemented with a RESTful web service. The system is designed to include different systems like hospital information system (HIS) via the RESTful web service very easily and without loss of data. The first prototype is implemented and will be expanded.
\end{abstract}

Keywords: surgical workflow, integrated operating room, workflow management system, BPM

\section{PURPOSE}

The operating room (OR) is a stressful working environment for all involved actors. Surgeons, scrub- nurses, OR-managers, cleaning persons or the hospital wards staff need to fulfill their tasks without mistakes and in time. The tasks of each team member in the OR need to be synchronized during a procedure even without one another knowing the task of the whole team. As of today, the OR staff benefits from their experience, using direct or telephone conversation to synchronize with other team members or even walk long distances within the facility to collect all needed information[1][2]. Often, the OR-manager is the person who calls all persons in charge, collecting data manually going around and asking each individual their status quo and figuring out how to handle everything efficiently in time. Organizational tasks often have to be fulfilled by clinical staff who are not qualified for these tasks. This leads to a lot of time pressure in patient care.

To help them to synchronize and work in the most efficient way and to allow situationadaptive assist systems in the peri-operative environment, we are developing a workflow management system that is based on industry standards to enable a rapid adoption in the medical domain. We built a communication tool to interact with the workflow engine and designed a way to formalize clinical pathways using the Object Management Group (OMG) [3] standards Business Process Model and Notation (BPMN), Decision Model and Notation $(\mathrm{DMN})$ and Case Management Model and Notation (CMMN).

Medical Imaging 2019: Image-Guided Procedures, Robotic Interventions, and Modeling, edited by Baowei Fei, Cristian A. Linte, Proc. of SPIE Vol. 10951, 1095127 - (c) 2019 SPIE CCC code: $1605-7422 / 19 / \$ 18 \cdot$ doi: $10.1117 / 12.2512724$ 


\section{METHODS}

We use the OMG standards BPMN, CMMN and DMN to model medical processes. CMMN allows us to use adaptive case management (ACM) techniques [4] to handle complex situations, which might appear during an intervention [5].

The workflow models shall be executed by a workflow engine. After an evaluation process [6] considering over 40 systems including IBM Business Process Manager, Signavio, Edorasware and the Camunda BPM workflow engine, which were chosen for a closer evaluation, we decided to use the Camunda BPM workflow engine [7] to deploy and run the model. The Camunda BPM Workflow Engine is a free to use Workflow Engine supporting BPMN, CMMN and DMN. We installed the workflow engine on an in-house server to run the models. By default, the engine was controlled via a website hosted by the engine and every completed activity of a process had to be confirmed manually using the web interface. As of today, the engine only interacts with RESTful interfaces - a standardized web architecture.

To connect other systems to the workflow engine and to have a better interaction with it, we implemented a communication middleware (for a first description see [8]) with interfaces, as well as control panels for the actors. Using the middleware, data can be sent and received between the workflow engine and other systems, like a Hospital Information System (HIS) or a situation recognition system. The data cannot only be transferred, but also extended with relevant content and sent to the identified person in charge via the preferred communication tool, as email, pager, phone call etc...

\subsection{Workflow Engine}

Camunda provides a "Tasklist" web application, which gives the user an overview and an option to control tasks assigned to them (Figure 1). Another web application is the Cockpit, which can be used to monitor workflow and decisions. The Admin web application is used to organize permissions, users or groups.

To integrate Camunda BPM Workflow Engine into your own business environment, Camunda offers a REST service. All interaction with the workflow engine can be handled via the REST service. You can start or delete process instances, complete task and many more. There is no need to use the given web application anymore.

Camunda offers as well an external Modeler to edit BPMN processes as well as DMN tables. 


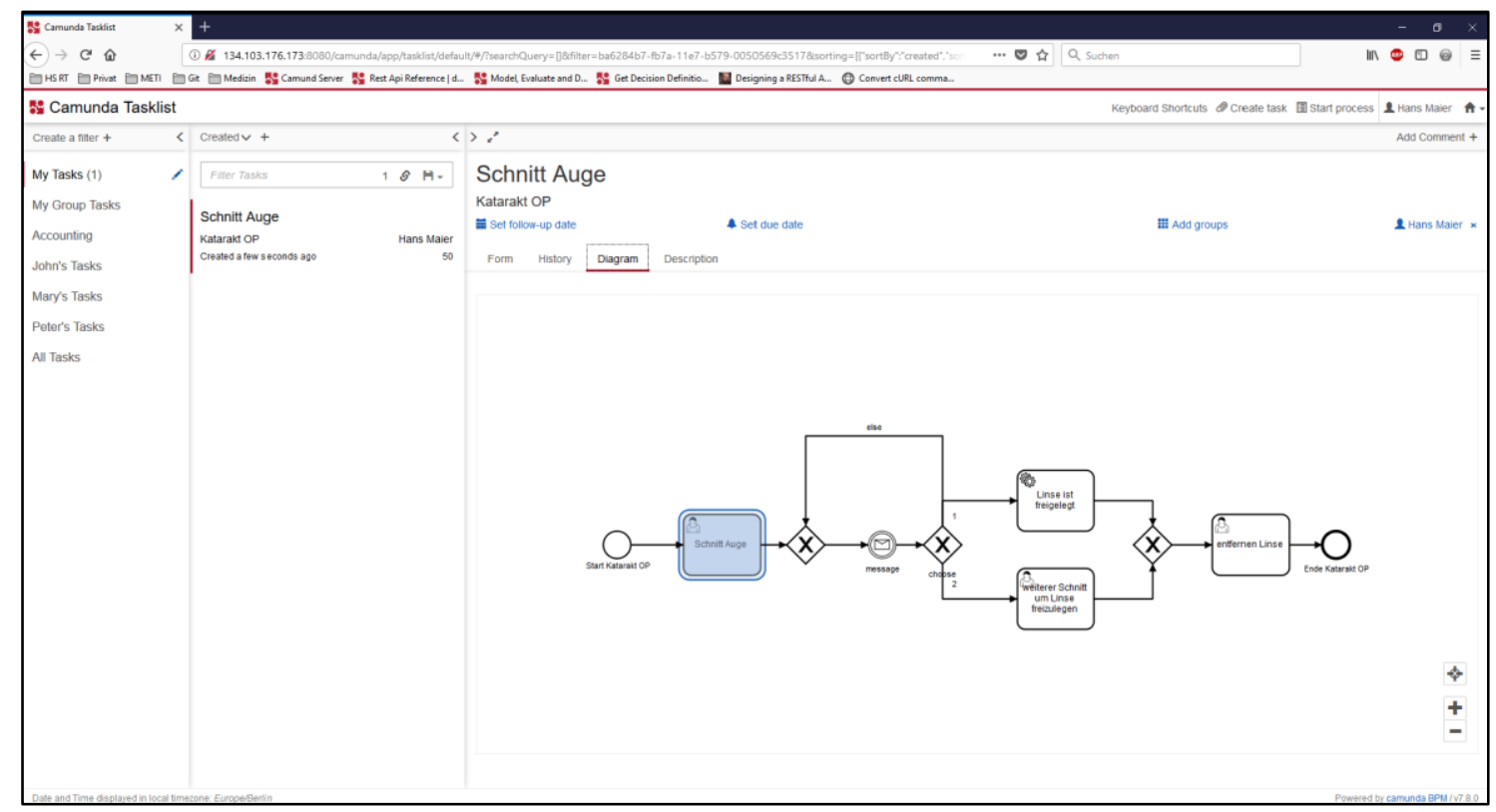

Figure 1. Camunda Tasklist web application

\subsection{Middleware}

The self-implemented middleware is written in python and has no graphical user interface. It offers a RESTful web service to interact with it (see Figure 2). Every interaction with the Camunda Workflow Engine is managed via the middleware. All sub-systems, like the control panels or the situation recognition system, need to send their queries or data to the middleware. The middleware analyzes the requests and forwards the data to the workflow engine. Information from the Workflow Engine to other systems is also sent through the middleware. All tasks are identified with a unique id, version, name and key. Every request to or from the middleware needs to provide this information to ensure that the correct task is addressed. The middleware verifies all requests to the workflow engine, if all information is provided and the sender is allowed to manipulate or get the data in case there is a need to collect more data e.g. from other systems. If all restrictions are met, the middleware sends the data to the workflow engine. Otherwise, the request will not be forwarded to the workflow engine and nothing happened. Using the middleware as a transmitter and translator gives the possibility to add systems with different protocols in future and to react to protocol changes of individual systems without changing the main interface to the Camunda engine. So, the workflow engine can be implemented easily in existing infrastructures only being connected via one interface to the middleware. 


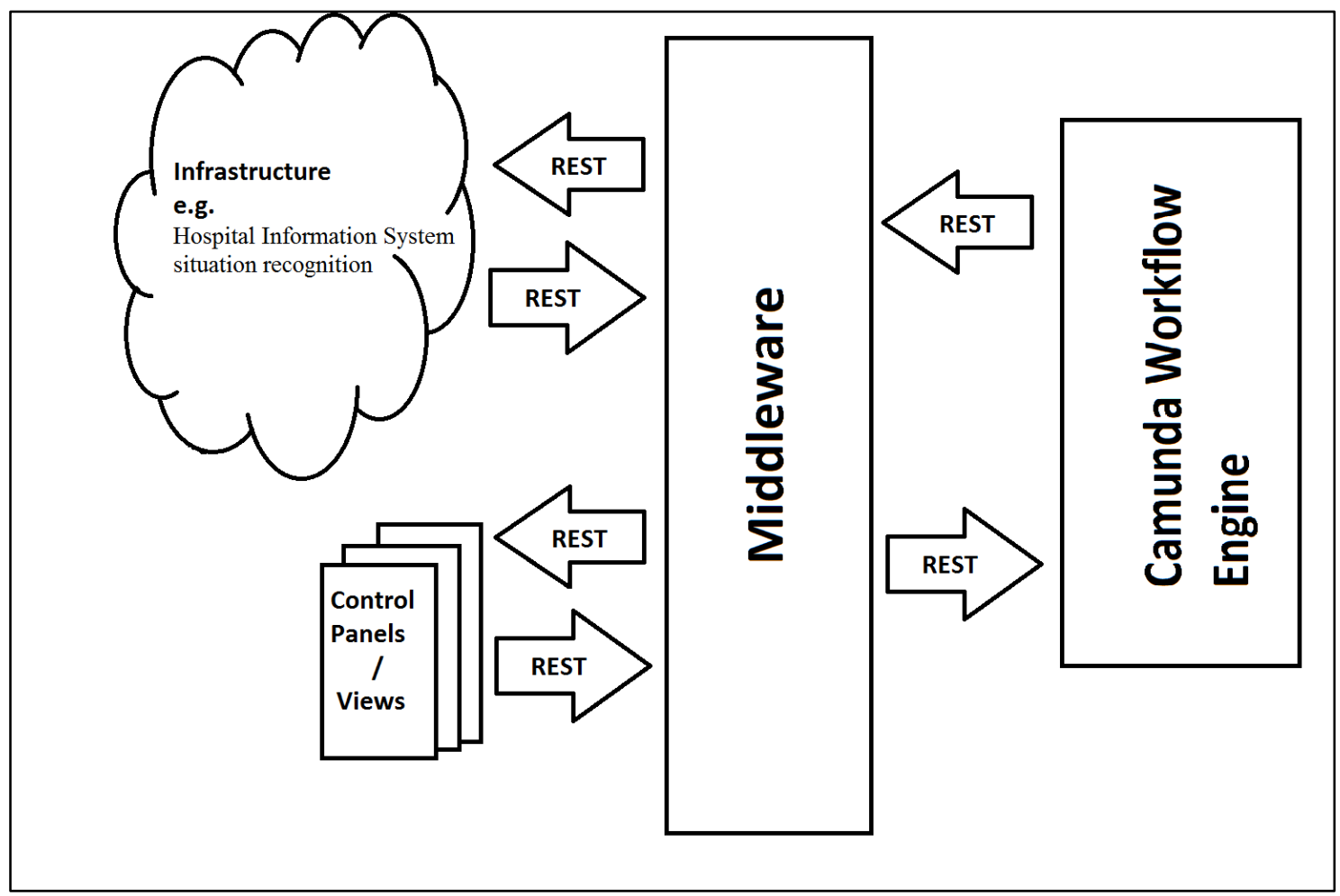

Figure 2. Workflow Management System

\subsection{Control Panels}

The control panels were used to display information and control the workflow engine. They are connected to the workflow engine via the middleware. You can load all deployed interventions, start a process instance of a deployed intervention, delete a process instance and interact with the process instance through runtime. Some functions of the control panel can and will be replaced later by other systems, for example the situation recognition. Currently a button simulates this. In Figure 3 is s screenshot of a control panel. 


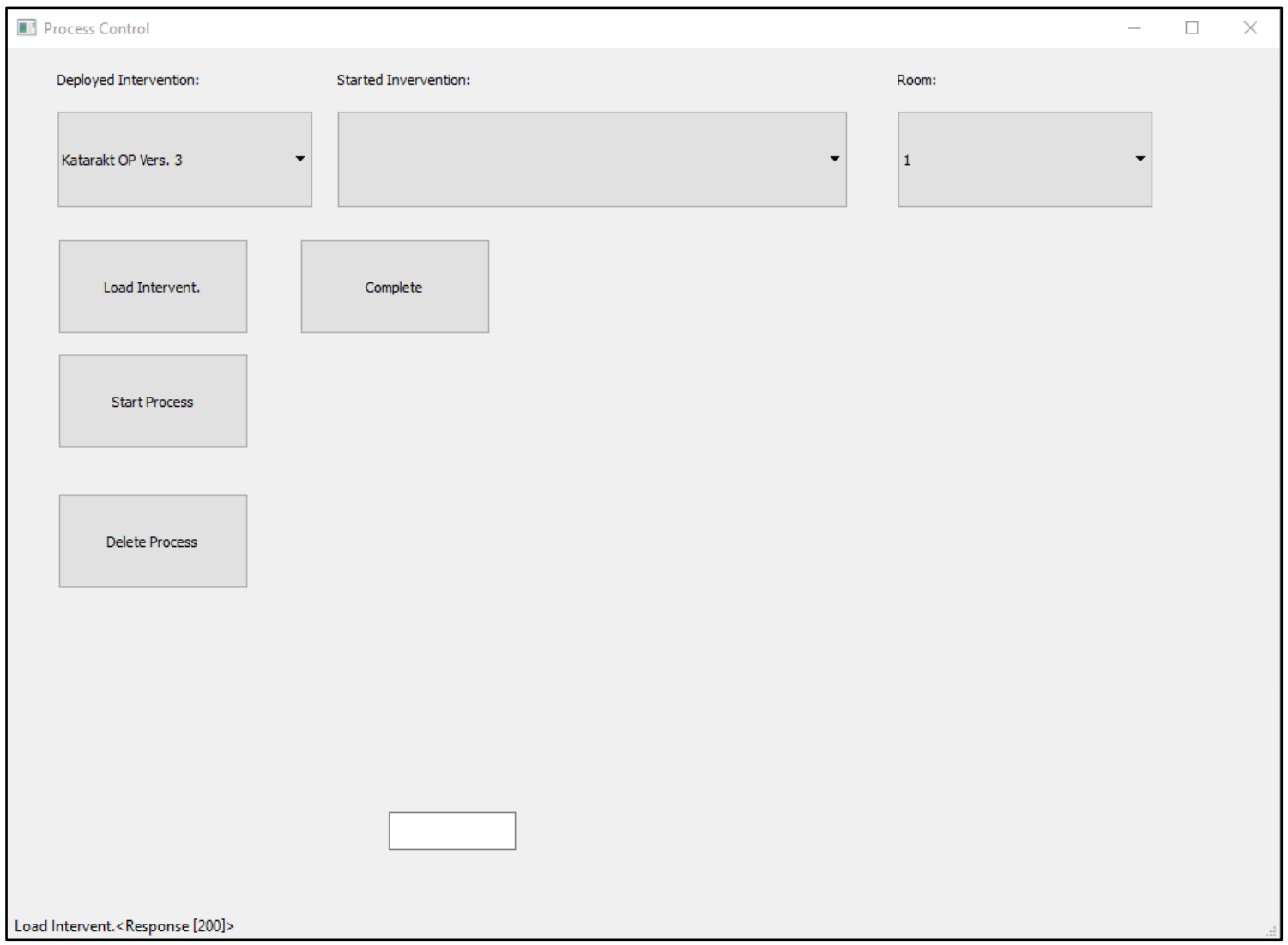

Figure 3. Process Control panel

\section{RESULTS}

We were able to model clinical pathways with OMG standards like BPMN, CMMN or DMN. Furthermore, we used the free Camunda BPM Workflow Engine to automatically run our modelled processes. The models and the workflow engine follow the OMG standard completely. By implementing a middleware, it offers a RESTful web service, to connect different systems to the workflow engine. This enables an easy implementation into existing infrastructure and easy adoption in future when interfaces of different systems or systems may change or may be added. We were able to interact with the workflow engine as well as to connect different software systems to the workflow engine like a control panel for the actors or a HIS. We modeled a cataract intervention using BPMN, CMMN and DMN [4] to run this model in our prototype.

\section{CONCLUSION}

The first prototype of the workflow management system is implemented. All OMG standards were implemented and demonstrated in this prototype. Now we need to record more data to test our system. The next step is to connect more systems to our prototype like the situation recognition to reduce the interaction for all actors. The system shall work more or less automatically without a lot of human input. Another step will be to model more clinical processes. 


\section{REFERENCES}

[1] Buzink, S. N., van Lier, L., de Hingh, Ignace H J T, and Jakimowicz, J. J. 2010. Risk-sensitive events during laparoscopic cholecystectomy: the influence of the integrated operating room and a preoperative checklist tool. Surgical endoscopy 24, 8, 1990-1995.

[2] Kohn, L. T., Corrigan, J., and Donaldson, M. S. 2000. To err is human. Building a safer health system. Quality chasm series. National Academy Press, Washington, D.C.

[3] The Object Management Group ${ }^{\circledR}\left(\mathrm{OMG}{ }^{\circledR}\right)$, Homepage https://www.omg.org/

[4] Wiemuth, M; Junger, D; Leitritz, MA; Neumann, J; Neumuth, T; Burgert, O (2017): Application fields for the new Object Management Group (OMG) Standards Case Management Model and Notation (CMMN) and Decision Management Notation (DMN) in the perioperative field., Int J Comput Assist Radiol Surg. 2017 Aug;12(8):1439-1449. doi: 10.1007/s11548-0171608-3. Epub 2017 May 18.

[5] Neumann, J; Wiemuth, M; Burgert, O; Neumuth, T (2017) Application of activity semantics and BPMN 2.0 in the generation and modeling of generic surgical process models, Journal of the International Foundation for Computer Assisted Radiology and Surgery: International Journal of Computer Assisted Radiology and Surgery Volume 12, p. 48 - 49, ISSN: 18616410, Springer Verlag

[6] Birinci, E (2017): Untersuchung einiger Workflow Engines auf deren Leistungsumfang im perioperativen Bereich, Bachelor Thesis, Reutlingen University, 21.03.2017, Supervisor: O. Burgert.

[7] Camunda Services GmbH, Homepage https://camunda.com/de/

[8] Wiemuth, M; Kroisant, A; Burgert, O (2017): Service-orientierte Enterprise Architektur zur chirurgischen Prozesssteuerung, 16. Jahrestagung der Deutschen Gesellschaft für Computerund Roboterassistierte Chirurgie (CURAC), p. 237-241, ISBN 978-3-95900-158-8, Hannover 